Supporting Information

for

\title{
Using desmotropes, cocrystals and salts for manipulating reactivity in mechanochemical organic reactions
}

by

Magdalena Kralj, Stipe Lukin, Goran Miletić, and Ivan Halasz*

Division of Physical Chemistry, Ruđer Bošković Institute, Bijenička 54, 10000 Zagreb, Croatia

E-mail: ivan.halasz@irb.hr 


\section{List of Figures}

S1 In situ Raman monitoring of neat milling of barb (in its keto form) and its transformation to the enol form of barbituric acid. ${ }^{1} \ldots \ldots \ldots \ldots$. . . . . . 1

S2 Rietveld plots for (top) the starting barbituric acid and (bottom) the enol form obtained after 90 minutes of neat grinding. . . . . . . . . . . . . . . S2

S3 Time-resolved in situ Raman spectra and corresponding Rietveld plots of the final products. . . . . . . . . . . . . . . . . S3

S4 Time-resolved in situ Raman spectra for the formation of the bm salt in a $\mathrm{LAG}(\mathrm{EtOH})$ reaction starting from barb and mel. . . . . . . . . . . . . . . . S4

S5 Rietveld plot for the product obtained in $\mathrm{LAG}(\mathrm{EtOH})$ of barb and mel. $\quad \ldots . . . \quad$. S4

S6 Time-resolved in situ Raman spectra for $\mathrm{NG}$ and $\mathrm{LAG}\left(\mathrm{H}_{2} \mathrm{O}\right)$ of barb and cya with the corresponding Rietveld plots. . . . . . . . . . . . . . . . . . . S5

S7 Rietveld plot for a sample extracted after 30 minutes of milling in the mixture of bm:van:cya. . . . . . . . . . . . . . . . . . . S6

S8 Rietveld plots for mo salt and bu cocrystal . . . . . . . . . . . . . . . . . S7

S9 Time-resolved in situ Raman spectra for reaction of barb and van with mel and ox. S8

S10 NMR spectrum in DMSO- $\mathrm{d}_{6}(400 \mathrm{MHz})$ of the raw reaction mixture obtained in the reaction of barb and van with a catalytic amount of mo. . . . . . . . . . . . . S9

S11 Time-resolved in situ Raman spectra for reaction of barb and van with urea and ox. S10

S12 Time-resolved in situ Raman spectra for reaction of barb and van with the uo cocrystal . . . . . . . . . . . . . . . . . . . S11

S13 Time-resolved in situ Raman spectra for $\mathrm{LAG}\left(\mathrm{H}_{2} \mathrm{O}, 2\right.$ eqv.) reaction in the 1:1:1:1 mixture of barb:van:mel:cya. . . . . . . . . . . . . . . . S11 


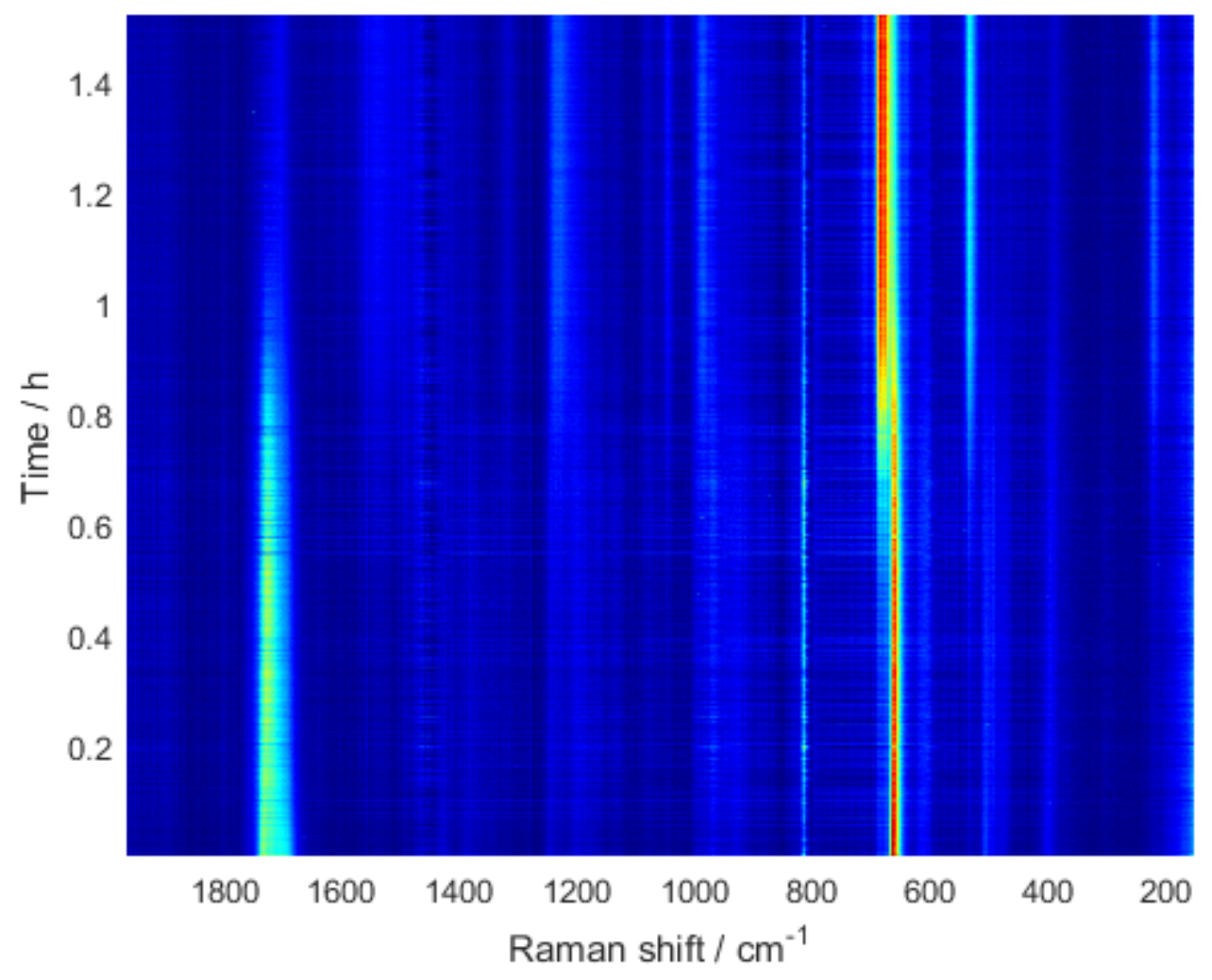

Figure S1: In situ Raman monitoring of neat milling of barb (in its keto form) and its transformation to the enol form of barbituric acid. ${ }^{1}$ 

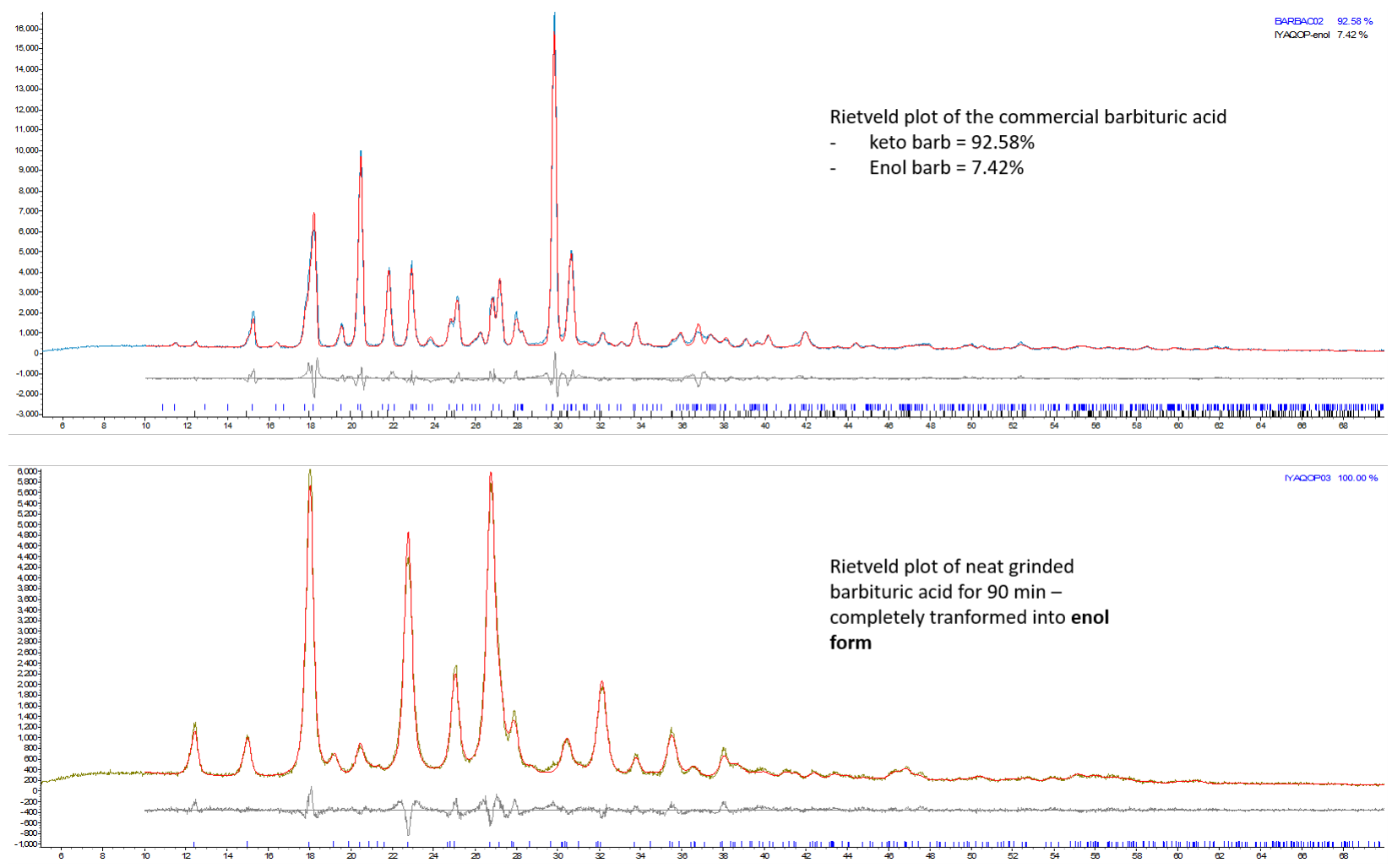

Figure S2: Rietveld plots for (top) the starting barbituric acid and (bottom) the enol form obtained after 90 minutes of neat grinding. 
barb:van

$n($ barb $): n($ van $)=1: 1$
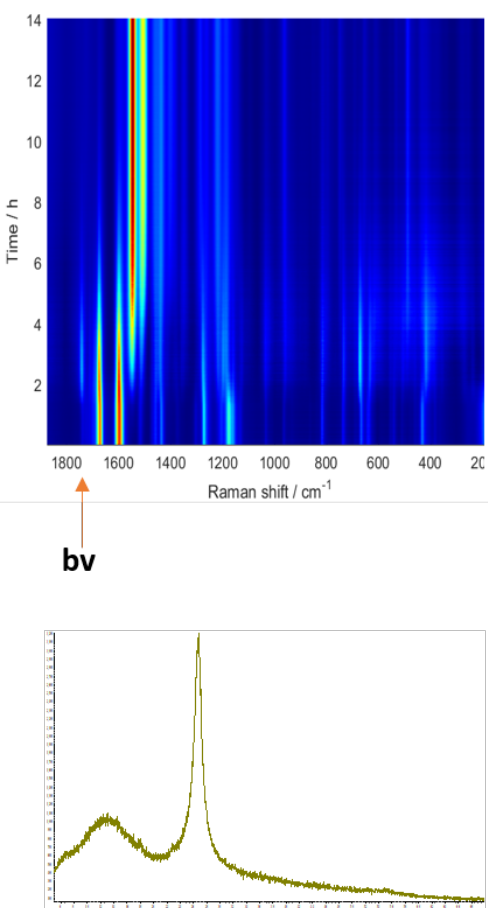

e-barb:van
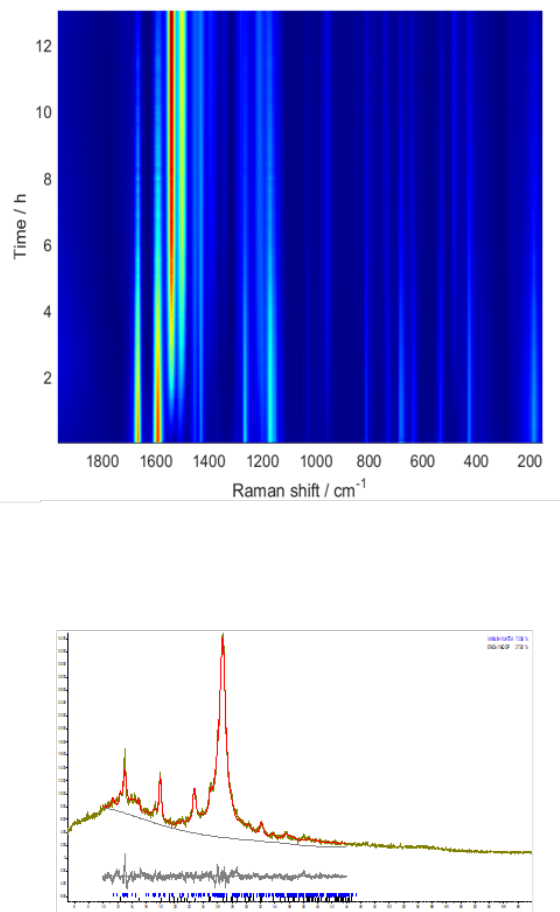

bm:van
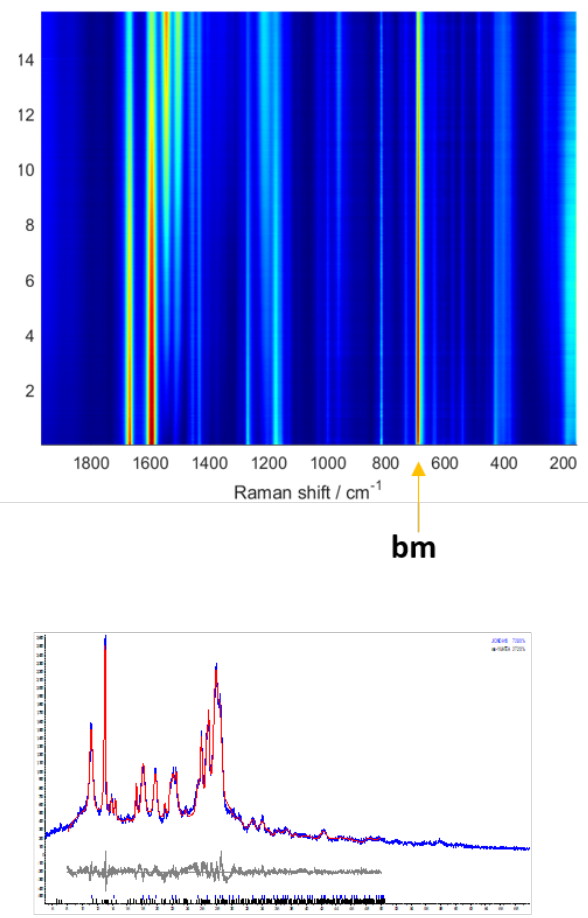

Figure S3: Time-resolved in situ Raman spectra and corresponding Rietveld plots of the final products. 


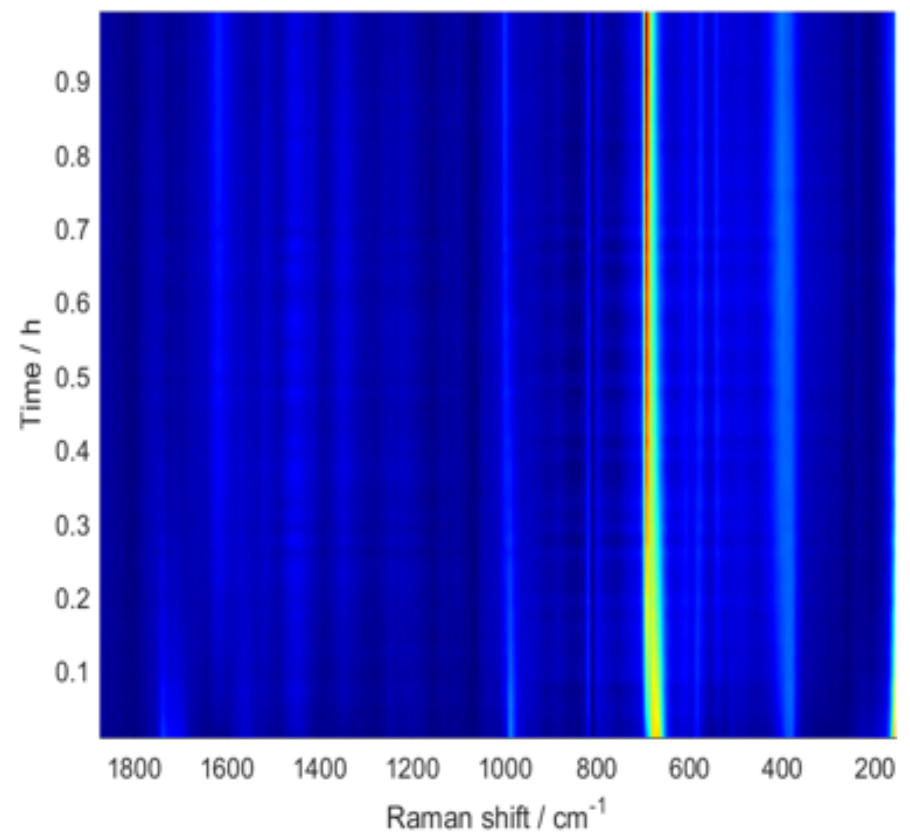

Figure S4: Time-resolved in situ Raman spectra for the formation of the bm salt in a $\mathrm{LAG}(\mathrm{EtOH})$ reaction starting from barb and mel.

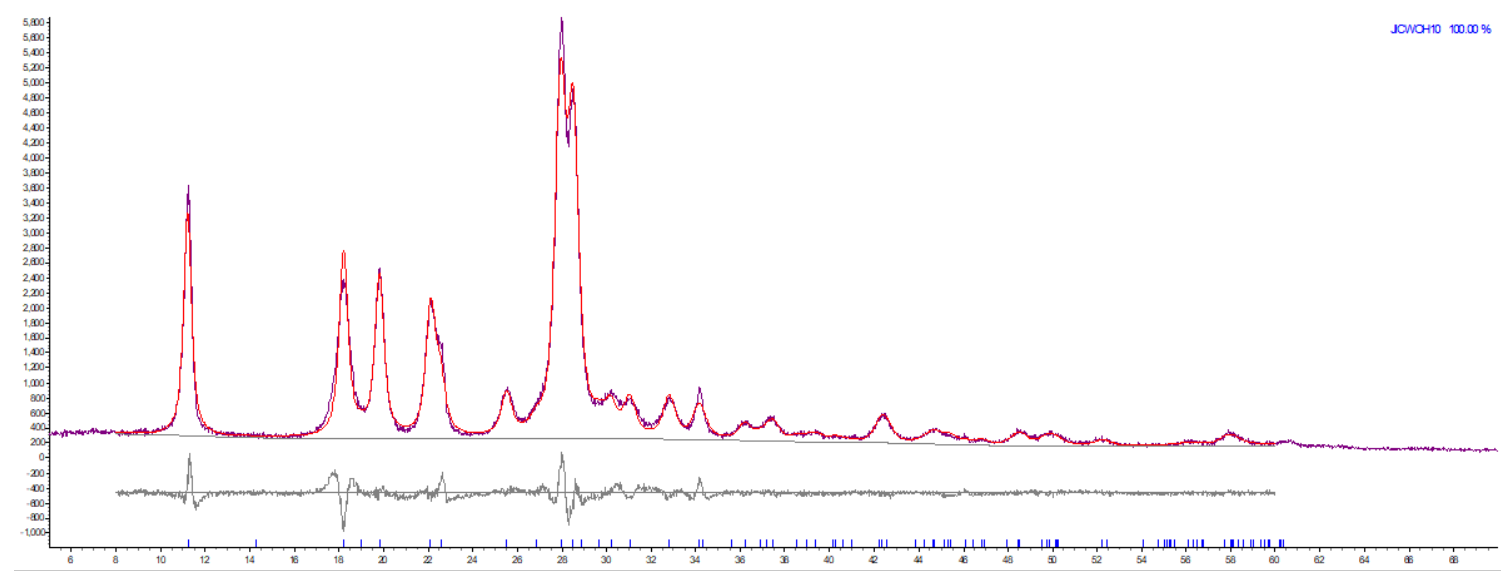

Figure S5: Rietveld plot for the product obtained in $\mathrm{LAG}(\mathrm{EtOH})$ of barb and mel. 
barb:cya = 1:1, NG
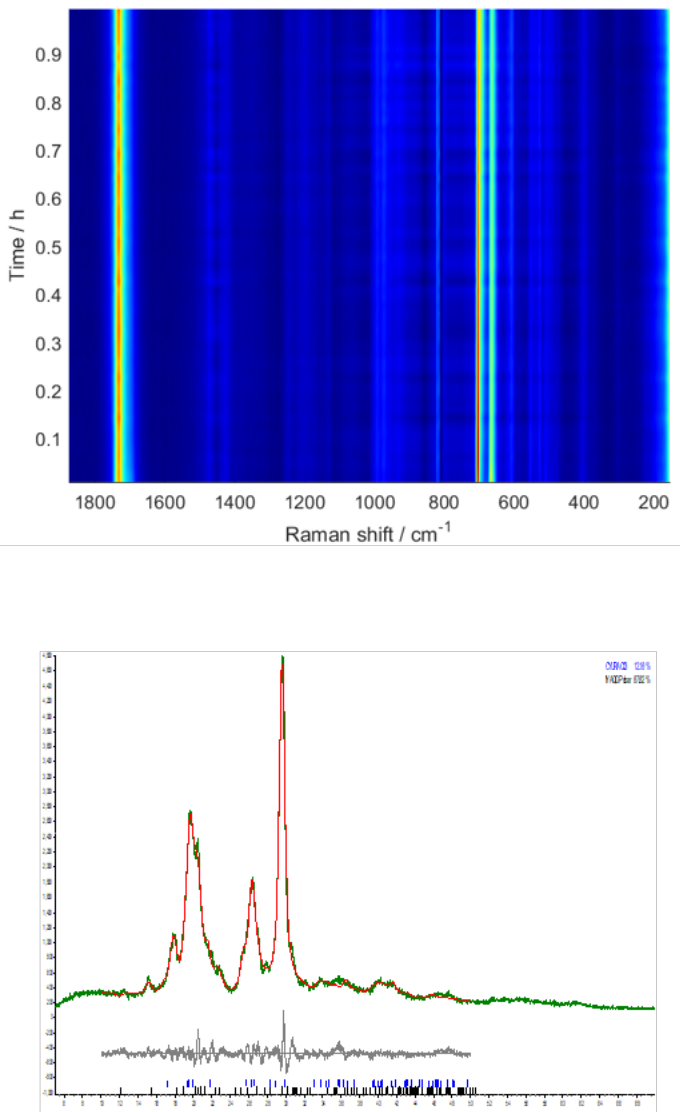

barb:cya = 1:1

LAG $(20 \mu \mathrm{L}$ EtOH)
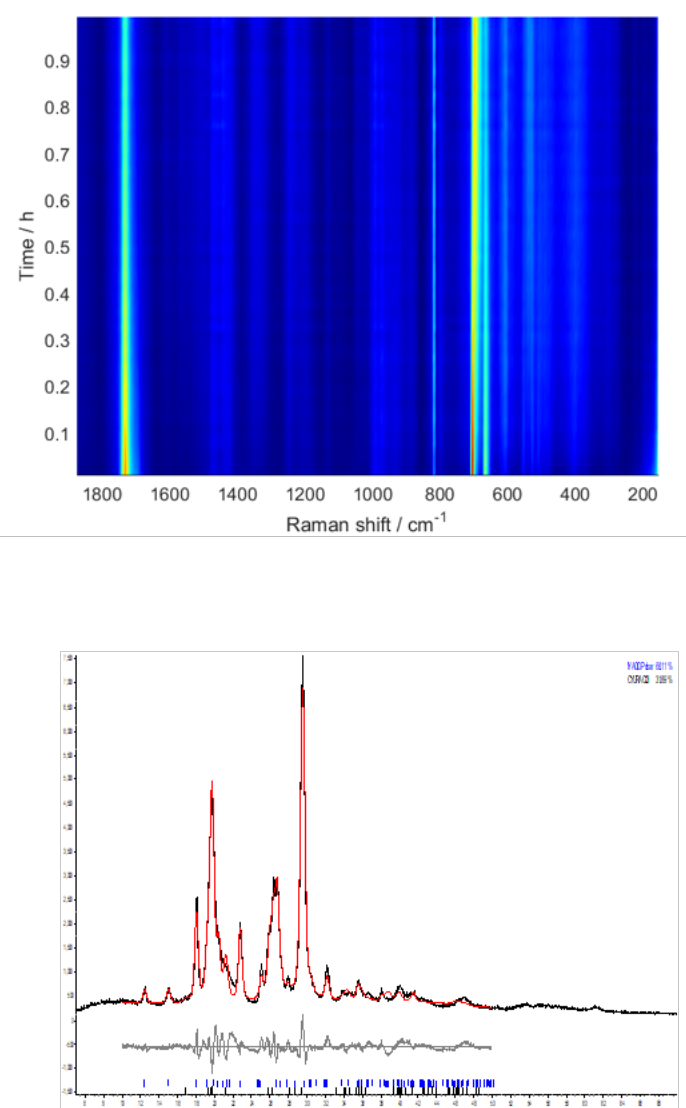

Figure S6: Time-resolved in situ Raman spectra for $\mathrm{NG}$ and $\mathrm{LAG}\left(\mathrm{H}_{2} \mathrm{O}\right)$ of barb and cya with the corresponding Rietveld plots for the products of milling given below. The mixtures remain physical mixtures and cya becomes poorly crystalline, particularly in NG. 


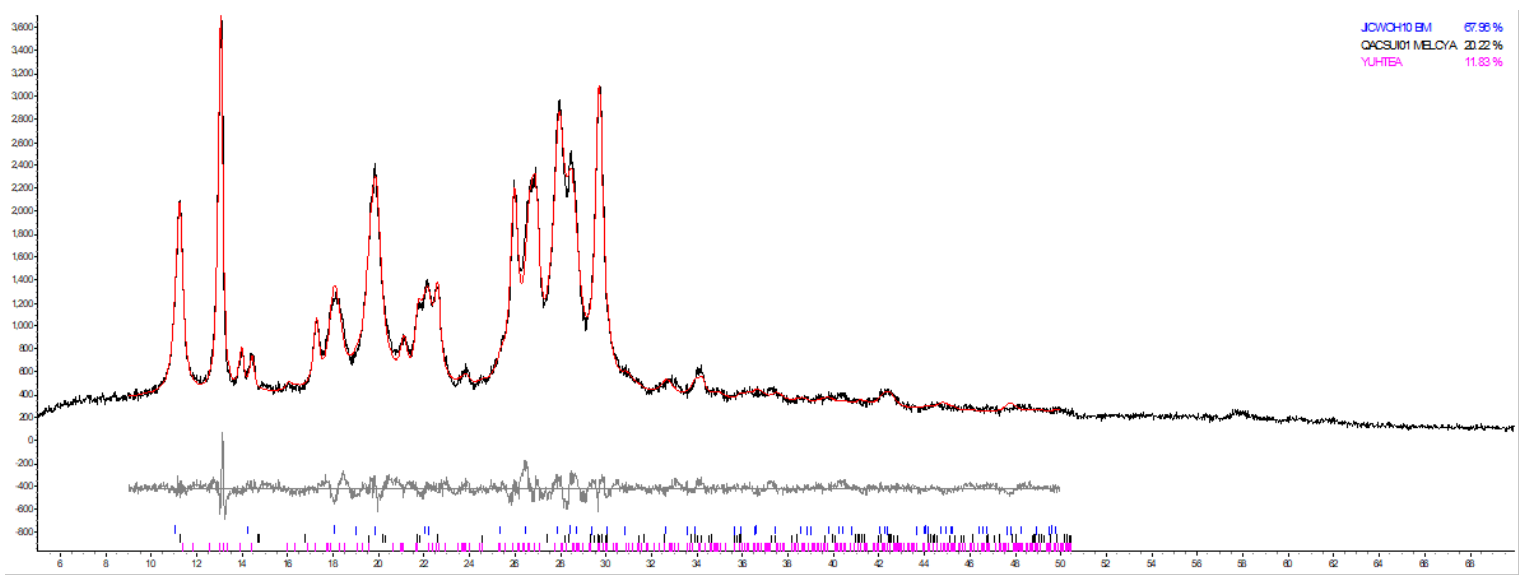

Figure S7: Rietveld plot for a sample extracted after 30 minutes of milling in the 1:1:1 mixture of bm:van:cya. the starting van (YUHTEA), bm (JICWOH10) and mc (QUACSUI01) are visible as crystalline phases since the product $\mathbf{1}$ forms amorphous. 

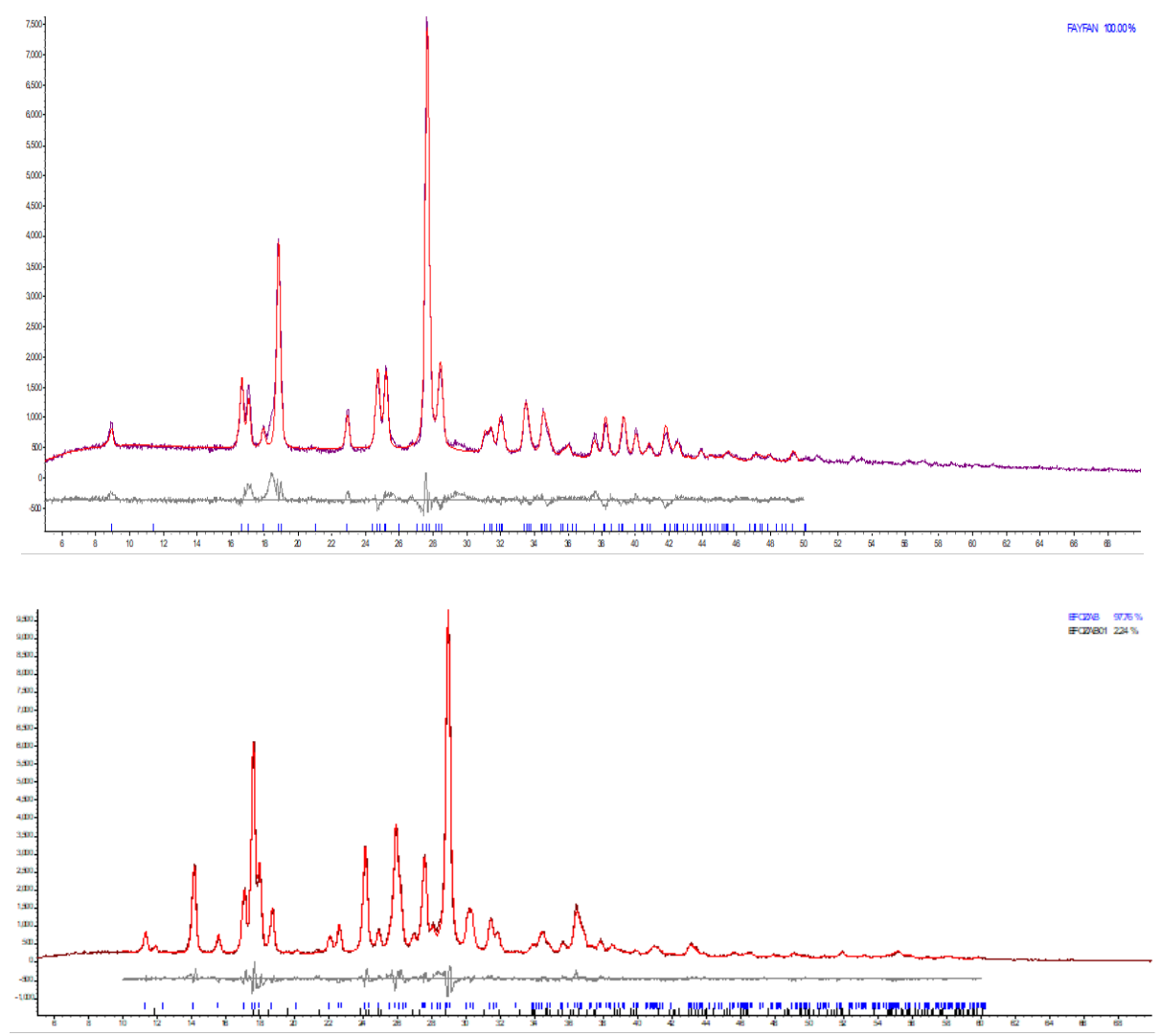

Figure S8: (top) Rietveld plot of the melamine-oxalic acid salt (CSD: FAYFAN) obtained after neat grinding of mel and $\mathbf{o x}$ for $30 \mathrm{~min}, 30 \mathrm{~Hz}$ and (bottom) Rietveld plot of the barbituric acid-urea cocrystal prepared by neat milling $(30 \mathrm{~Hz}, 60 \mathrm{~min})$ corresponding to a mixture of two polymorphs: $\operatorname{EFOZAB}\left(P 2_{1} / c\right)$ and EFOZAB01 $(C c)$. 

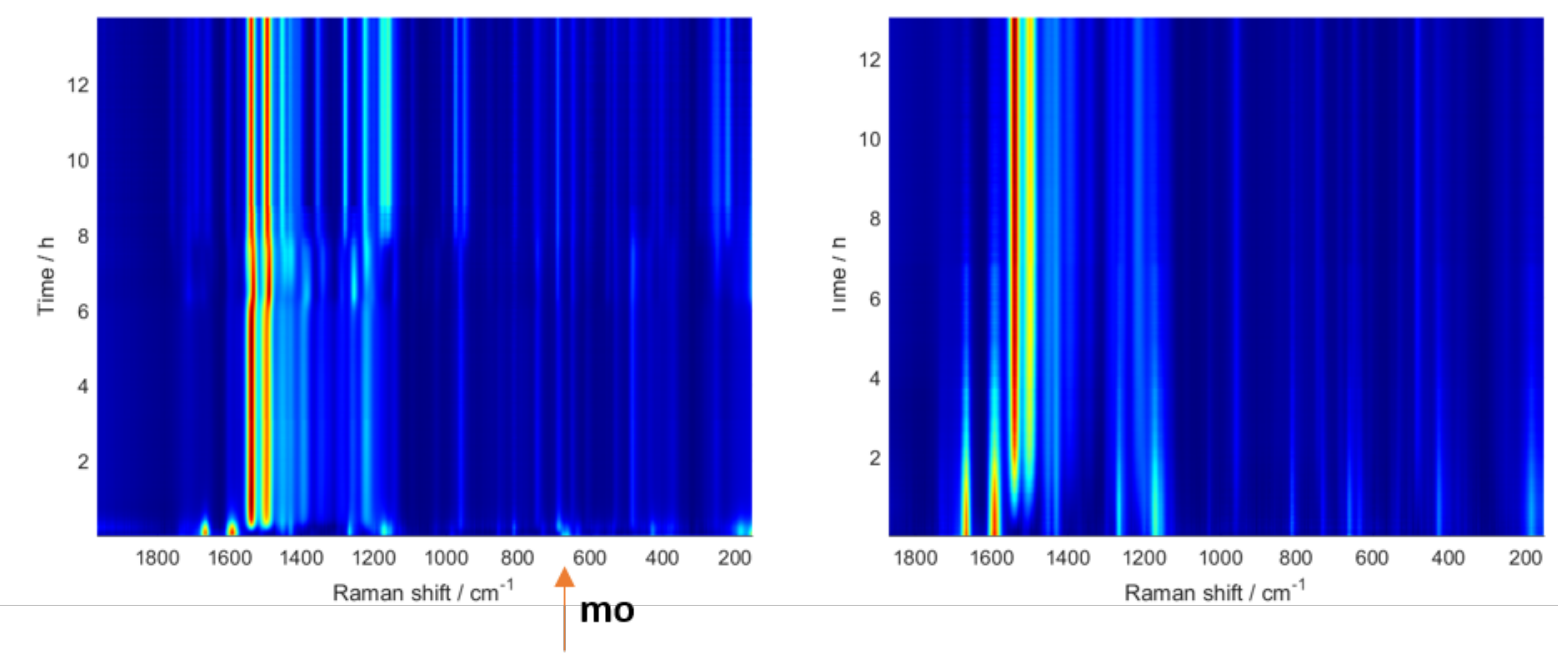

barb:van:mox $=1: 1: 1$

barb:van:mox $=1: 1: 0.1$
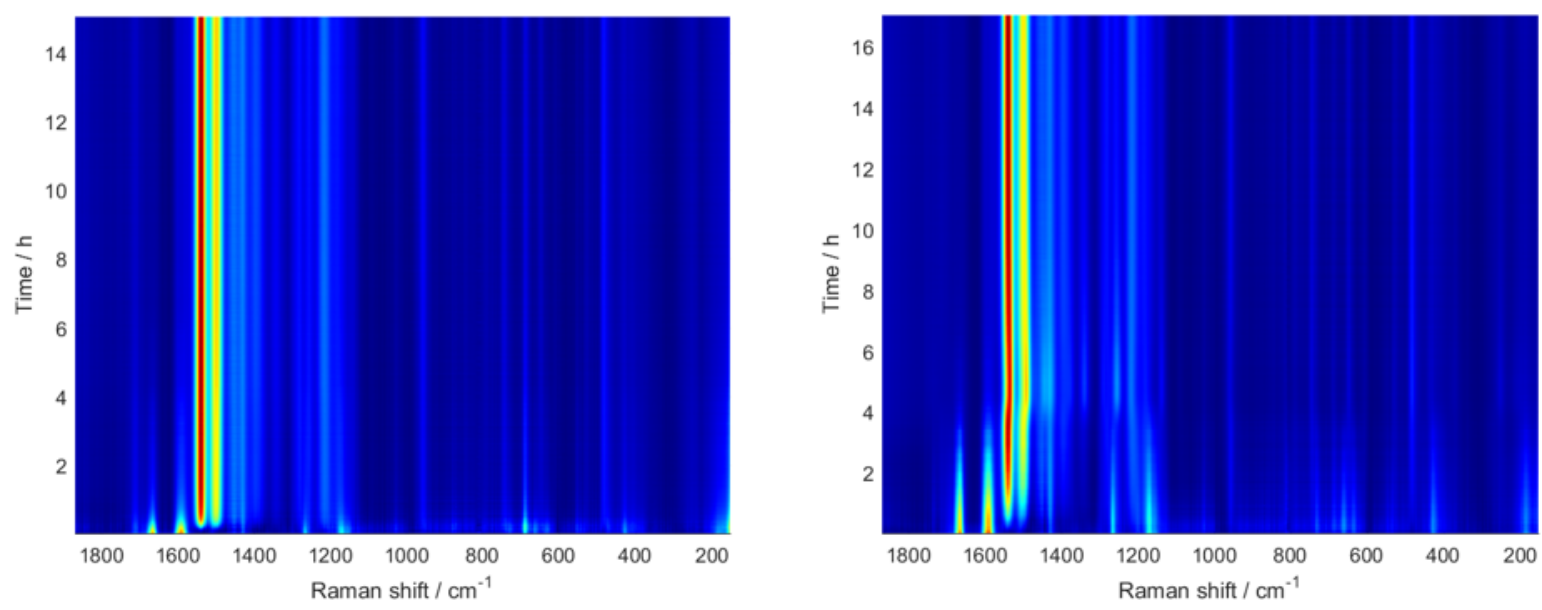

Figure S9: Time-resolved in situ Raman spectra for reaction of barb and van with mel and ox. (top left) Initially formed Form I is transforming into Form II after $6 \mathrm{~h}$ and to Form III around 8 $\mathrm{h}$. This transformation is usually not happening when mo is used, possibly due to less water being present since $\mathbf{m o}$ is anhydrous and $\mathbf{o x}$ is a dihydrate. 


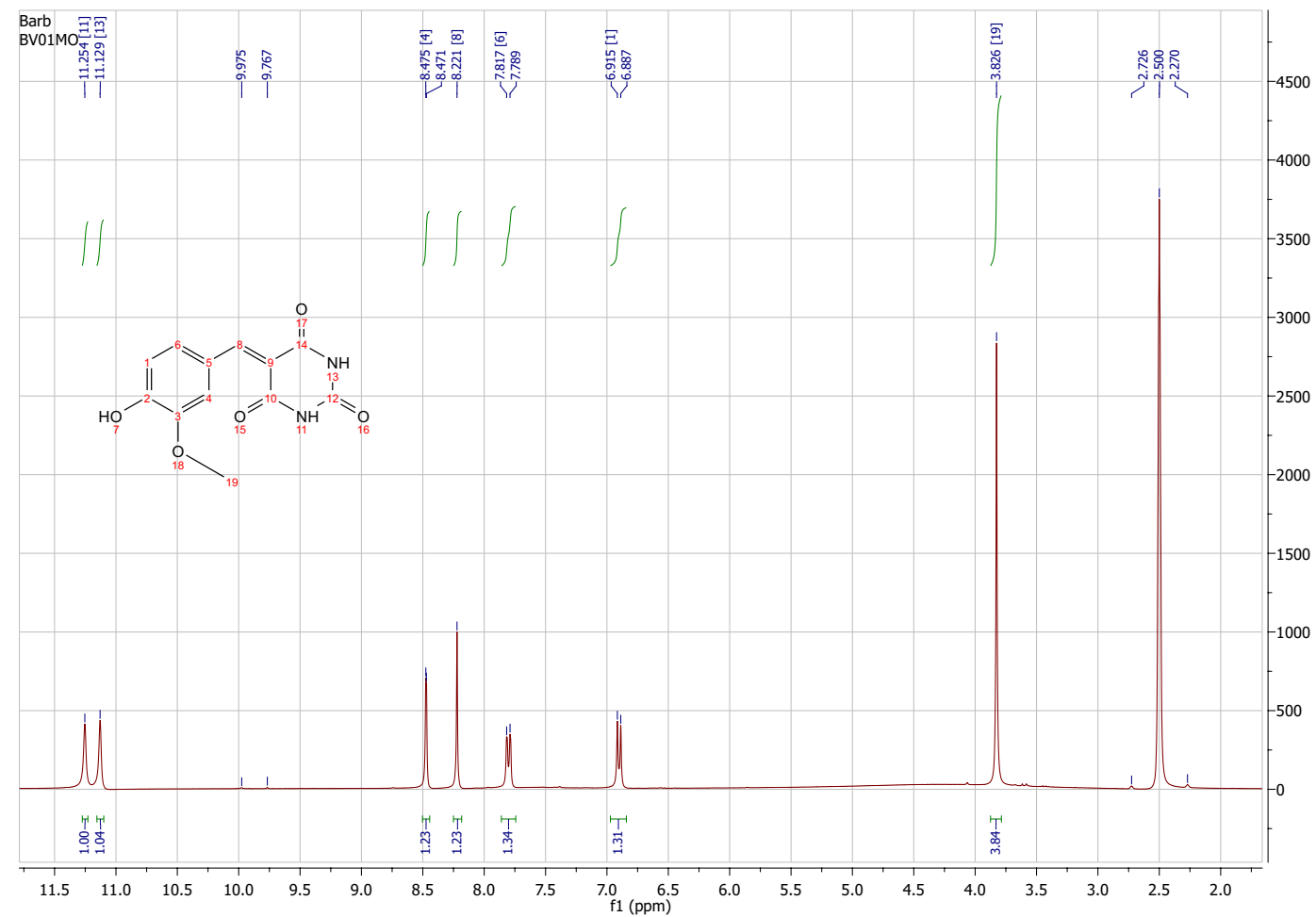

Figure S10: NMR spectrum in $\mathrm{DMSO}_{-} \mathrm{d}_{6}(400 \mathrm{MHz})$ of the raw reaction mixture obtained in the reaction of barb and van with a catalytic amount of mo. The spectrum shows a pure product 1. ${ }^{1} \mathrm{H}$ NMR (DMSO-d 6 , 400 MHz, d / ppm): 11.25 (s, 1H, H11), 11.13 (s, 1H, H13), 8.47 (d, 1H, H4), 8.22 (s, 1H, H8), 7.82 (d, 1H, H6), 6.92 (d, 1H, H1), 3.83 (s, 3H, H19). The peak at 3.83 ppm belonging to three methyl group protons is higher then expected due this peak sitting on a broad hump from water. 

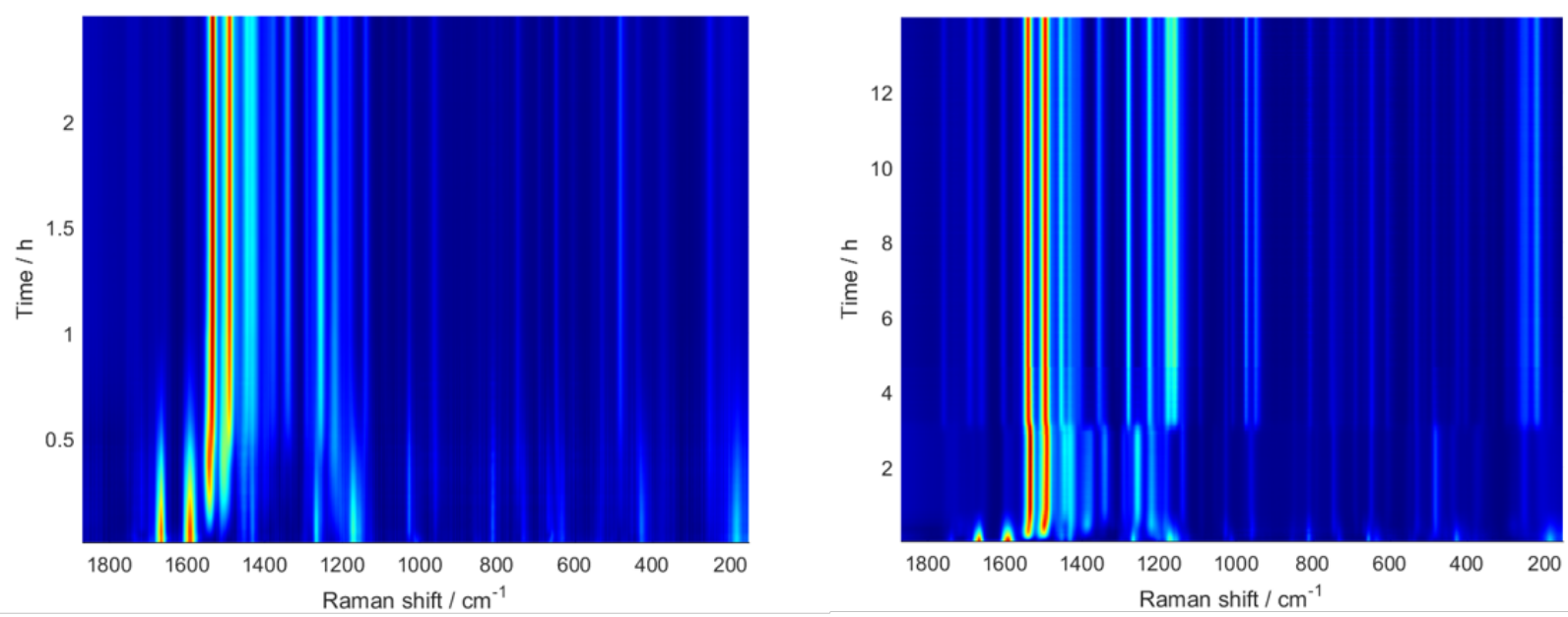

bu:van $=1: 1$

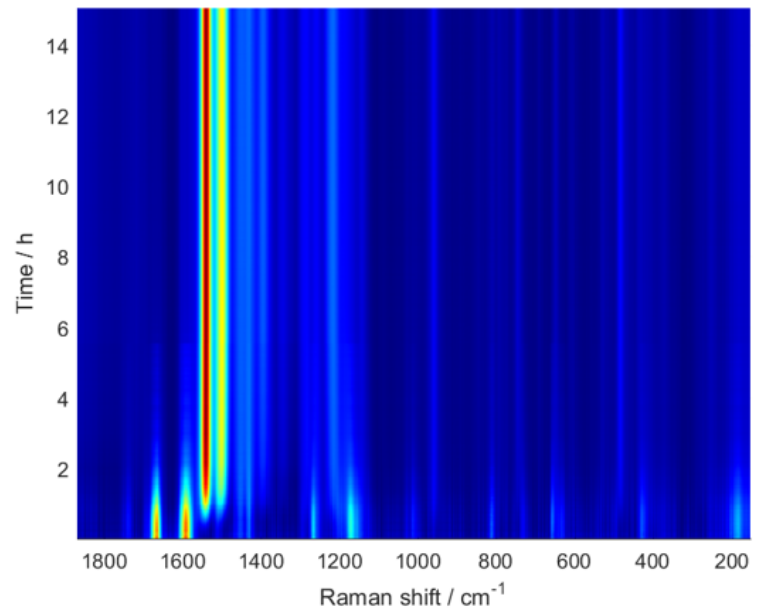

Figure S11: Time-resolved in situ Raman spectra for reaction of barb and van with urea and ox. The four component mixture: barb + van + urea + ox ends is Form II. Initially formed Form I in the reaction of bu $+\mathbf{v a n}+\mathbf{o x}$ is almost immediately transforming into Form II and to Form III after around $3 \mathrm{~h}$. Reaction of barb and van yields Form I. 

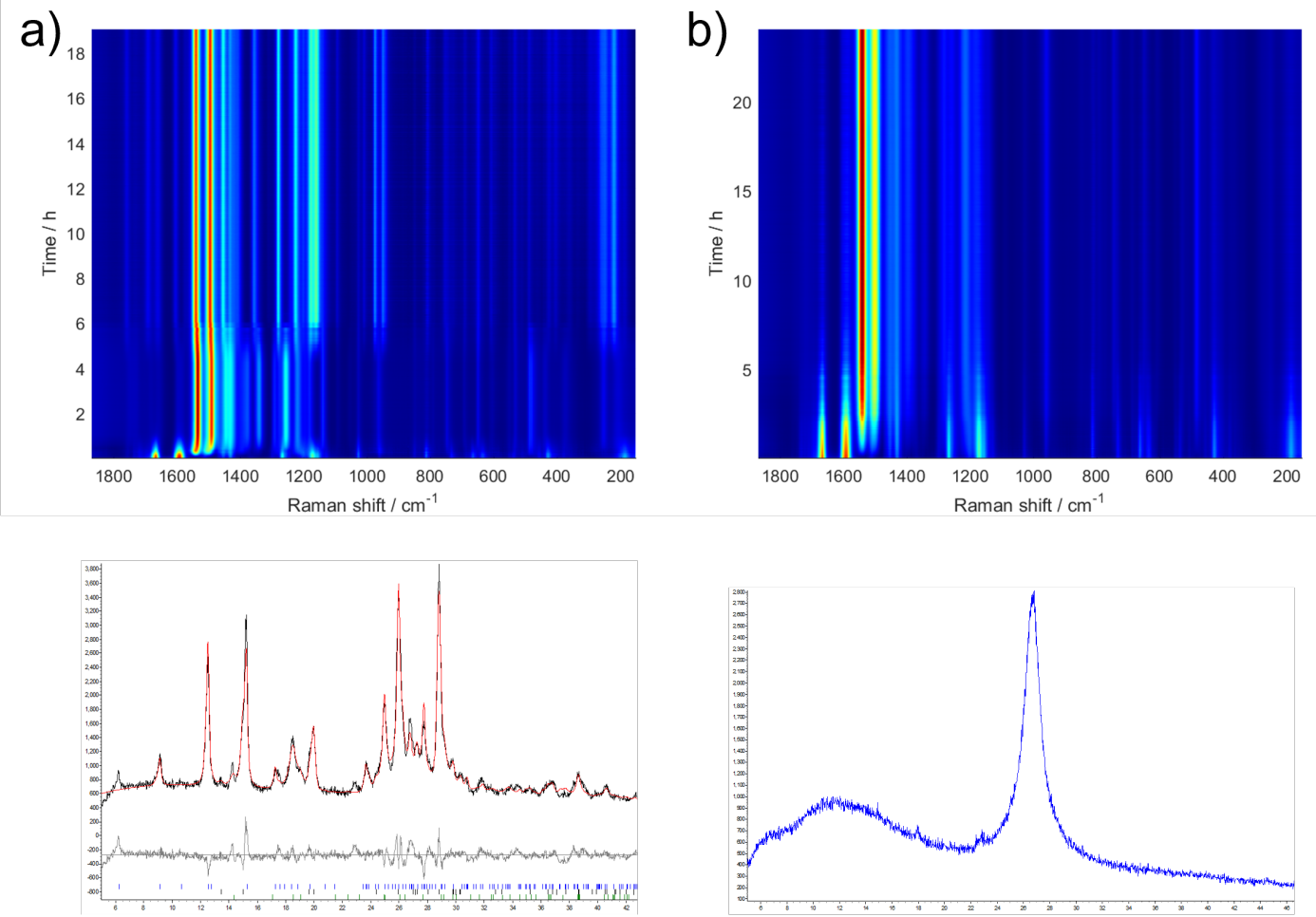

Figure S12: Time-resolved in situ Raman spectra for reaction of barb and van with the uo cocrystal in (a) equimolar amount and (b) in the amount of $10 \mathrm{~mol} \%$. Diffraction patterns of the corresponding product mixtures are given below 2D spectra. With equimolar amount of uo there is a sequence of transformations from Form I to Form III of $\mathbf{1}$, while if $10 \mathrm{~mol} \%$ of $\mathbf{u o}$ is used, the initially formed Form I (which is X-ray amorphous) remains stable.

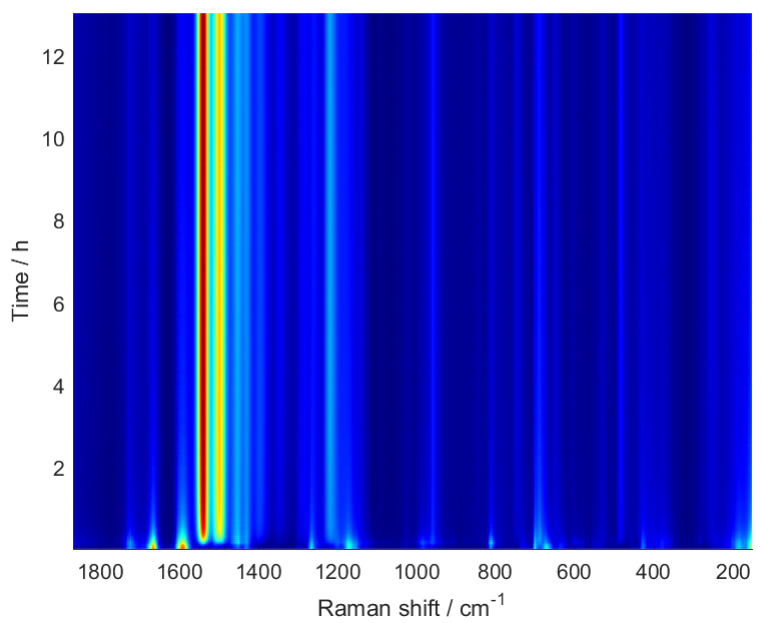

Figure S13: Time-resolved in situ Raman spectra for $\mathrm{LAG}\left(\mathrm{H}_{2} \mathrm{O}, 2\right.$ eqv.) reaction in the 1:1:1:1 mixture of barb:van:mel:cya. 


\section{References}

[1] Schmidt, M. U.; Brüning, J.; Glinnemann, J.; Hützler, M. W.; Mörschel, P.; Ivashevskaya, S. N.; van de Streek, J.; Braga, D.; Maini, L.; Chierotti, M. R.; Gobetto, R. The Thermodynamically Stable Form of Solid Barbituric Acid: The Enol Tautomer. Angew. Chem. Int. Ed. 2011, 50, 7924-7926. 